\title{
MICROMECHANICAL PROPERTIES AND STRESS MEASUREMENTS WITH DIFFRACTION METHODS
}

\author{
Elżbieta Gadalińska ${ }^{1}$ \\ Andrzej Baczmański \\ ${ }^{1}$ Institute of Aviation, Warsaw, Poland \\ ${ }^{2}$ AGH-University of Science and Technology, Kraków, Poland
}

\begin{abstract}
Diffraction methods are commonly used for the determination of the elastic lattice deformation and distortion from the displacement and broadening of the diffraction peak. These methods enable researchers to measure stresses and elastic properties of polycrystalline materials. The main advantages of diffraction methods are their non-destructive character and the possibility of macrostress and microstress analysis for multiphase and anisotropic materials. Measurements are performed selectively only for crystallites contributing to the measured diffraction peak, i.e. for the grains having lattice orientations for which the Bragg condition is satisfied. When several phases are present in the sample, measurements of separate diffraction peaks allow for the behaviour of each phase to be investigated independently. This method can be applied without any limitations to flat specimens.

Numerical calculations of residual stresses around the rivets imply a very high stress gradientin the case of tangential stresses as well in the case of radial stresses. Attempting to verify these predictions, the residual stress measurements with an $X$-ray diffractometer were performed on riveted samples after the riveting process. In addition, complementary measurements of strain values with strain gauges during the riveting process were performed as well as the finite elements modelling. The aim of these measurements was to determine the stress values around the rivets and to compare results obtained with different techniques.

On the other hand, the multi-scale crystallographic model of elastoplastic deformation is very convenient for the study of elastoplastic properties in microscopic and macroscopic scales. Comparison of experimental data with model predictions allows us to understand the physical phenomena that occur during a sample's deformation at the level of polycrystalline grains. Moreover, the micro and macro parameters of elastoplastic deformation can be experimentally established. It should be stated that the characterisation of the residual stress field and elastic properties is important in the study of the mechanical behaviour of polycrystalline materials, including plasticity and damage phenomena.

In this work, a new analysis method of neutron diffraction results obtained during in-situ tensile load is proposed and tested. The methodology is based on the measurements of lattice strains during in-situ tensile testing for several hkl reflections and for different orientations of the sample with respect to the scattering vector. As the result, the full stress tensor for preferred texture orientations in function of the applied stress can be determined using the crystallite group method. The experimental data are presented and compared with the self-consistent model calculations performed for groups of grains selected by different hkl reflections.
\end{abstract}

Keywords: X-ray diffraction, stress measurements on riveted samples, duplex steel, composites, stress measurements during in-situ tensile test, stress concentarion tensor, necking phenomenon. 


\section{GENERAL RULES OF METHODOLOGY OF STRAIN AND STRESS MEASUREMENTS PERFORMED WITH DIFFRACTION METHODS}

\subsection{Stress in polycrystalline materials - classification}

Residual, or internal stress, is a type of stress caused by persistent strain which remained in the material for example after the treatment. This kind of stress can be defined as a derivate of elastic strains remaining after technological treatment and processing of the material. Residual stresses remain in the material after lightening the specimen (e.g. in the case of plastic deformation). Residual stresses remain in the material when the surface of the specimen was deformed to a considerably bigger extent than the inner part of the specimen. This type of stress can be also observed in the case of the two-phase composites due to a different thermal expansion coefficient of each individual composite phase. In this case, in each phase a different strain appears. The other reason for the appearance of residual stresses is the laser treatment of the surface.

Depending on the stress changes vs. distance there are three types of residual stresses:

- First Order Stresses: they represent the constant part for a relatively big proportion of the specimen, for a large quantity of grains. Values of First Order Stresses do not depend on the position of the measurement point. Normally, First Order Stresses appear in the rolled specimen where a stress component parallel and perpendicular for the rolling direction appears (Fig. 1a).

- Second Order Stresses: they vary from grain to grain. Second Order Stresses can be observed in a rolled specimen, too. They originate from different plastic deformations of an individual grain. Average in the whole volume of the specimen for stresses of this kind is zero (Fig. 1b).

- Third Order Stresses: their values vary inside every individual grain. They describe the stress field originating from dislocations or grain boundaries. The average value of Third Order Stresses in the whole volume of the grain is zero (Fig. 1c).

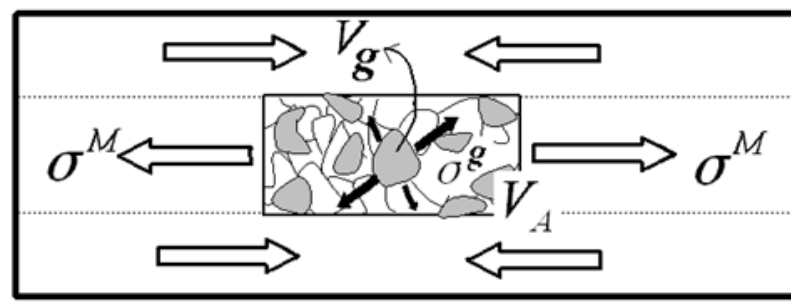

$$
\bigcirc p h_{1} \in V_{p h 1} \quad D p h_{2} \in V_{p h 2}
$$

a)

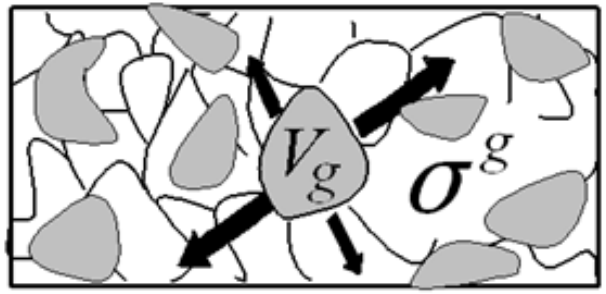

b)

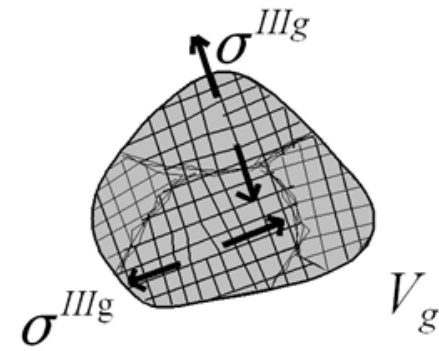

c) 
Fig. 1. Stress clasification: a) first order stresses, b) second order stresses, c) third order stresses.

\subsection{The effect of residual strain and stress on the diffraction image}

First and Second Order Stresses cause a systematic shift of the diffraction peaks visible in the intensity vs. $2 \Theta$ graph (Fig. 2). Diffraction peaks originate from many grains so the information about the measured strain is averaged information. It is important to note that not every grain gives diffraction peaks, but only these with an appropriate orientation and which meet Braggs' law. Third Order Stresses originate from dislocations of the atoms (atoms are not placed in their nodes) (Fig. 3a). Thrid Order stresses cause the broadening of the diffraction peaks (Fig. 3b). The same phenomenon appears for specimens made of materials with different grain sizes. There are some methods (Sherrer's or Williamson-Hall 's formulas) which enable calculating the grain size from the diffraction peak broadening.

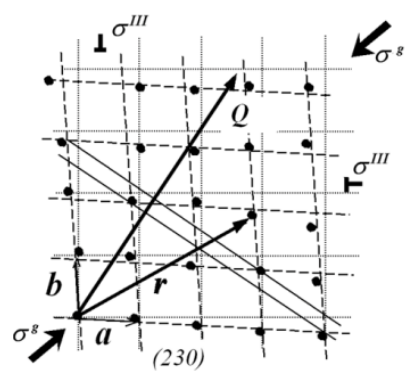

a)

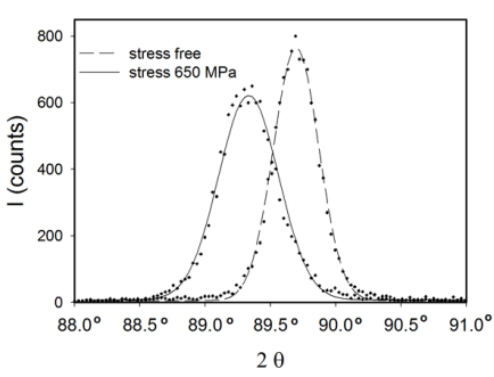

b)

Fig. 2. Deformation of the cystal lattice in case of first and second order stresses (a) and its influence on the position of diffraction peaks $(b)$.

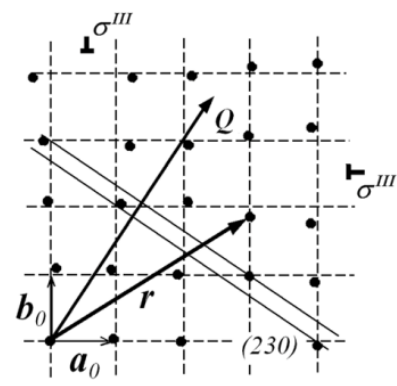

a)

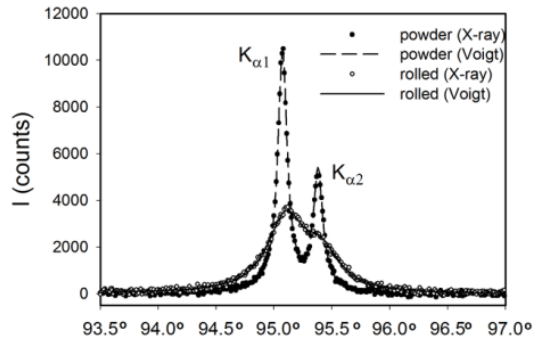

$2 \theta$

b)

Fig. 3. Deformation of the cystal lattice in case of third order stresses (a) and its influence on the broadening of diffraction peaks (b).

Strain values one is able to obtain employing he Braggs' law (eq. 1):

$$
n \lambda=2 d_{h k l} \sin \Theta
$$

Where:

$n$ - natural number,

$\lambda$ - wavelength of the $\mathrm{X}$ radiation used in the experiment,

$d_{h k \digamma}$ inter-planar distance for the specimen after deformation,

$\Theta$ - Bragg's angle.

And the definition of the strain (eq. 2): 


$$
\langle\varepsilon\rangle_{h k l}=\frac{\langle d\rangle_{h k l}-d_{h k l}^{0}}{d_{h k l}^{0}}
$$

Where:

$\langle\varepsilon\rangle_{h k l}$ - average strain for grains taking part in diffraction, for $h k l$ planes, for deformed material,

$\langle d\rangle_{h k l}$ - average inter-planar distances for $h k l$ planes, for deformed material,

$d_{h k l}^{0}$ - inter-planar distances for $h k l$ planes, for undeformed material

Figure 4 shows the relation between the change of inter-planar distances and the value of Bragg's angle. When the specimen is stretched, the inter-planar distances grow (Figs. 4a,b). This causes Braggs' angle to decrease and the diffraction peak to shift (Fig. 4c).

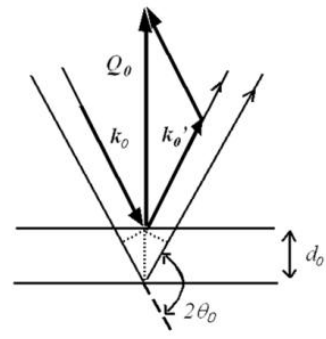

a)

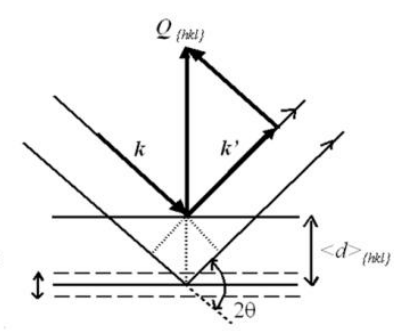

b)

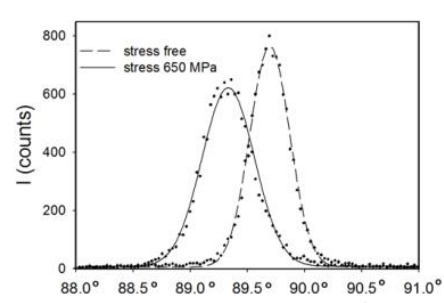

$2 \theta$

Fig. 4. Relation between the change of inter-planar distances and the value of the Braggs' angle.

It is very important to notice that the diffraction occurs only for grains for which the scattering vector if perpendicular for $h k l$ planes (the darkest grains on the fig. 5).

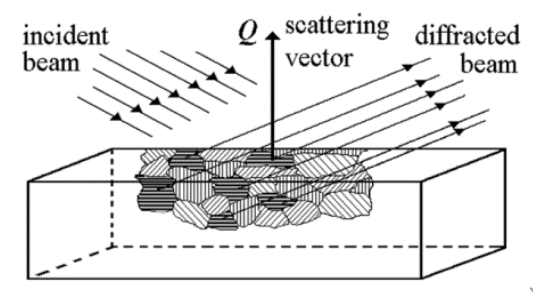

a)

Fig. 5. X-ray difffraction for grains which hkl planes are perpendicular to the scattering vector.

After measuring the strain values for distinguished grains the stress values can be obtained with the following equation:

$$
\langle\varepsilon(\varphi, \psi)\rangle_{h k l}=F_{i j}(h k l, \varphi, \psi) \sigma_{i j}^{I}
$$

Where:

$\varepsilon(\varphi, \psi)_{\mathrm{hkl}}$ - the average value of strain for $h k l$ planes, direction defined by $\varphi$ and $\psi$ angles, $\mathrm{F}_{\mathrm{ij}}(\mathrm{hkl}, \varphi, \psi)$ - diffraction elastic constants for $h k l$ planes, direction defined by $\varphi$ and $\psi$ angles; values which depend on the Miller indices $h k l$ and the $\varphi$ and $\psi$ angles. Values of diffraction elastic constants can be known as a result of the appropriate experiment or modeling,

$\sigma_{i j}^{I}-$ First Order Stress values,

$\varphi$ - angle between the direction of measured strains and the preferable axis of the specimen (see Fig. 6), first angle which defines the orientation of the scattering vector,

$\psi$ - angle between the scattering vector and the perpendicular to the specimen surface (see Fig. 6), second angle which defines the orientation of the scattering vector. 


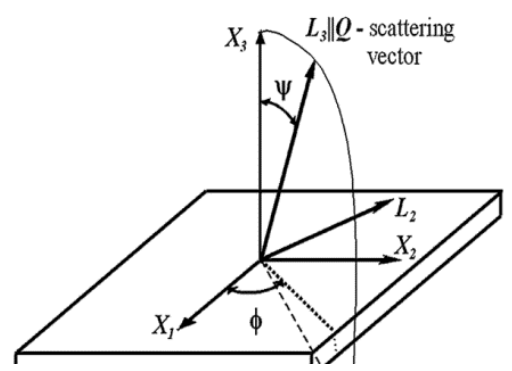

Fig. 6. Definition of $\varphi$ and $\psi$ angles.

The simplest case is the case of the specimen without texture. It is the base of the most commonly used technique employing $\mathrm{X}$ radiation. In this case, only two elastic constants, $\mathrm{s}_{1}$ and $\mathrm{s}_{2}$, are independent. The linear regression of the experimental points to the function $\langle d(\varphi, \psi)\rangle_{h k l}$ vs. $\sin ^{2} \psi$ (fig. 7) gives information about the stress value in a given direction:

$$
\langle d(\varphi, \psi)\rangle_{h k l}=\frac{1}{2} s_{2} d_{h k l}^{0} \sigma_{11} \sin ^{2} \psi+s_{1} d_{h k l}^{0}\left(\sigma_{11}+\sigma_{22}\right)+d_{h k l}^{0}
$$

Where:

$\langle d(\varphi, \psi)\rangle_{h k l}$ - average inter-planar distances for $h k l$ planes and for $\varphi, \psi$ angles, for deformed material,

$s_{1}, s_{2}$ - elastic constants,

$\sigma_{11}, \sigma_{22}-$ stresses in given directions.

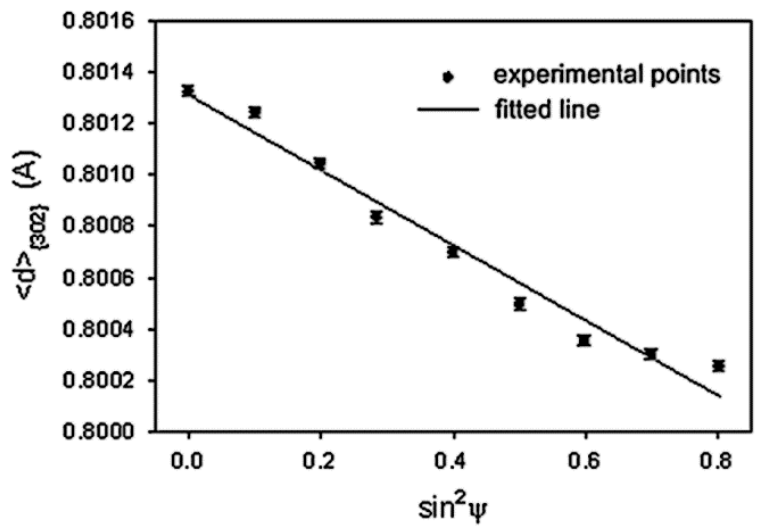

Fig. 7. Linear regression on the graph $\langle d(\varphi, \psi)\rangle_{h k l}$. vs. $\sin ^{2} \psi$

The slope $(a)$ of the fitted line is a result of the linear regression which gives information about the stress value in given direction. It is obtained with the following equation:

$$
\begin{gathered}
a=\frac{1}{2} s_{2} \sigma_{11} d_{h k l}^{0} \\
\sigma_{11}=\frac{2 a}{s_{2} d_{h k l}^{0}}
\end{gathered}
$$




\section{MEASUREMENT TECHNIQUES EMPLOYING DIFFERENT RADIATION TYPES}

\subsection{Penetration depth and shape of the examined material volume for different types of radiation}

While measuring strain one can employ three types of radiation: $\mathrm{X}$, neutron and synchrotron radiation. Measurements with $\mathrm{X}$ radiation are the most popular in engineering applications because of its accessibility. Measurements with every individual type of radiation vary with respect to the geometry, penetration depth, shape and volume of the examined material.

- X-radiation: the penetration depth is relatively small - about several micrometers depending on the material, the type of X-radiation and the angle between the incident beam and the specimen surface. This type of radiation enables measurements of stress values on the surface only - the assumption is that the stress component perpendicular to the specimen surface equals zero (Fig. 5). - Neutron radiation: the penetration depth is bigger than in the case of $\mathrm{X}$-radiation, it can be even few centimeters (Table 1). Typically, the examined volume is a cube which dimension is about $1 \mathrm{~mm}$ or more (Fig. 8).

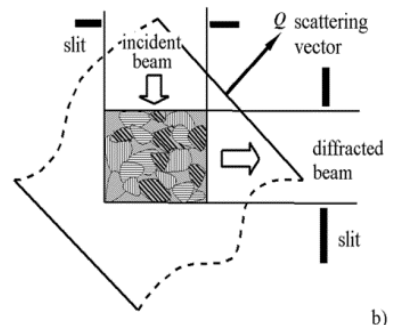

Fig. 8. Examined volume and ist shape for neutron radiation.

- Synchrotron radiation: the penetration depth is about few millimeters, centimeters and the measured volume is diamond shape whose width can be even a few micrometers (fig. 9). It allows perform strain measurements considerably faster than in case of $X$ radiation and with a better resolution.

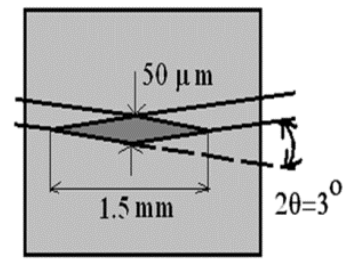

Fig. 9. Examined volume and ist shape for synchrotron radiation.

Table 1. Penetration depth for different types of radiation.

\begin{tabular}{|c|c|c|c|}
\cline { 2 - 4 } \multicolumn{1}{c|}{} & $\begin{array}{c}\text { NEUTRONS } \\
\lambda=1.29 \AA(E \approx 50 \mathrm{meV})\end{array}$ & $\begin{array}{c}\text { X- RAY } \\
\lambda=1.54 \AA \mathrm{A} \mathrm{AK} \alpha(\mathrm{E} \approx 8 \mathrm{keV})\end{array}$ & $\begin{array}{c}\text { SYNCHROTRON } \\
\lambda=0.15 \AA \quad(E \approx 80 \mathrm{keV})\end{array}$ \\
\cline { 1 - 4 } ELEMENT & $(\mathrm{mm})$ & $(\mathrm{mm})$ & $(\mathrm{mm})$ \\
$\mathrm{yl}$ & 70.5 & $0.5 \times 10^{-1}$ & 15 \\
$\mathrm{Fe}$ & 6.2 & $0.29 \times 10^{-2}$ & 1.8 \\
$\mathrm{Ni}$ & 3.7 & $0.17 \times 10^{-2}$ & 1.4 \\
$\mathrm{~W}$ & 6.6 & $0.21 \times 10^{-2}$ & 1 \\
\hline
\end{tabular}




\subsection{The measurement geometries}

While employing $\mathrm{X}$ or neutron radiation one can use one of two most commonly used geometries:

- $\psi$ geometry: the diffraction plane (the plane described by the scattering vector, incident and diffracted beams) is tilted toward the specimen surface (fig. 10),

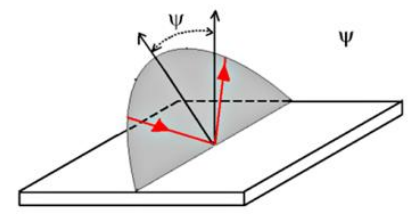

Fig. 10. The \& geometry.

- $\omega$ geometry: the diffraction plane is perpendicular to the specimen surface, the scattering vector is tilted toward the specimen surface in the diffraction plane (fig. 11).

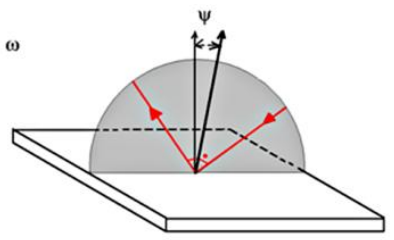

Fig. 11. The w geometry.

The most popular mode for synchrotron radiation is the transmission mode (fig. 12). The Debeye-Sherrer rings are observed on a two-dimensional detector. Sometimes, the transmission mode is employed in case of neutron radiation, too.
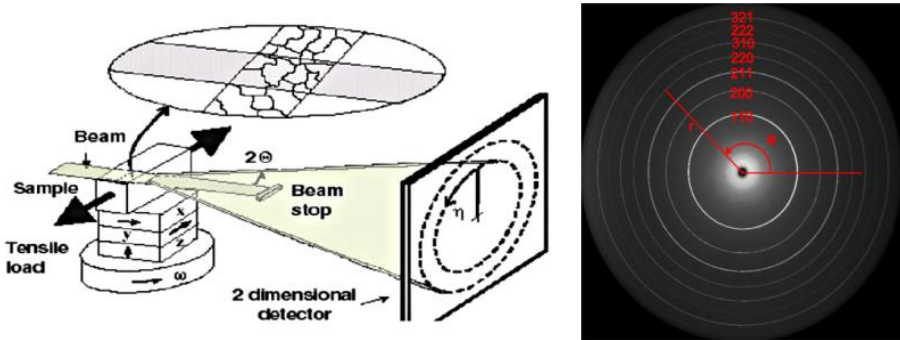

Fig. 12. Transmission mode for synchrotron radiation.

\subsection{Neutron diffraction - spallation source and time-of-flight method (TOF).}

A special technique is employed in the measurements with neutron diffraction. This technique uses the spallation source of neutrons. The production of neutrons at a spallation source begins with a high-powered accelerator. The accelerator produces an intense beam of protons focused onto a target (uranium, tantalum). Inside the target, spallation processes produce neutrons, initially at very high energies. These neutrons are then slowed in moderators filled with liquid hydrogen or liquid methane down to the energies that are needed for the scattering instruments.

The spallation source produces neutrons of different energies (different velocities). These neutrons diffract on the crystal lattice of the specimen material according to Bragg's law. The dispersion appears. Information about the neutron wavelength can be obtained from the de Broglie equation and the connection with Bragg's law gives the relation between the time of flight and the inter-planar spacings. 


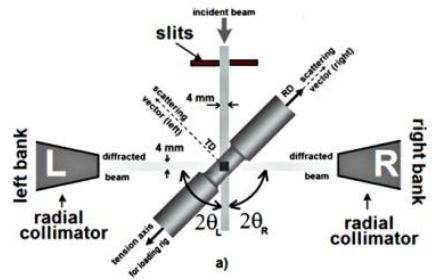

Fig. 13. Neutron diffraction measurement geometry with TOF method.

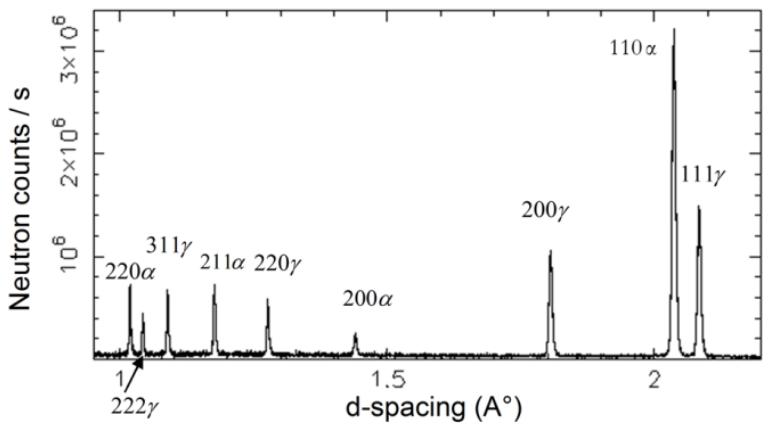

Fig. 14. Diffraction pattern for TOF method.

\section{STATE-OF-THE-ART: CHOSEN EXPERIMENTS}

\subsection{The effect of thermal treatment on the mechanical behaviour of duplex steel}

The plastic behaviour of phases was studied in an UR45 sample subjected to different treatments, i.e. quenching and aging after annealing at $1050^{\circ} \mathrm{C}$. In this case, the neutron diffraction was used to measure in situ the lattice strains in both phases under an applied tensile load. In this case, an experimental setup similar to ENGIN $X$ was used. In this case, however, only one diffraction peak was measured for each phase by the $2 \theta$ dispersion method at G5.2 spectrometer (LLB, Saclay, France). The incident neutron wavelength of $2.87 \AA$ was used to obtain scattering angles from austenite (111) and ferrite (110) peaks at $2 \theta \approx 86^{\circ}$ and $2 \theta \approx 90^{\circ}$, respectively. Again, the model results were adjusted to the lattice strains in both phases and to the macro-mechanical curves (Fig. 15) [1]. It was found that the yield stress of the ferritic phase is higher in the aged material in comparison with the quenched one, while the yield stress of the austenite phase is the same in both samples. It means that it was only the hardness of Ferrite that increased during aging because of spinodal decomposition, while the hardness of austenite remained unchanged. As a consequence, the macroscopic yield stress also increased when the material was aged.
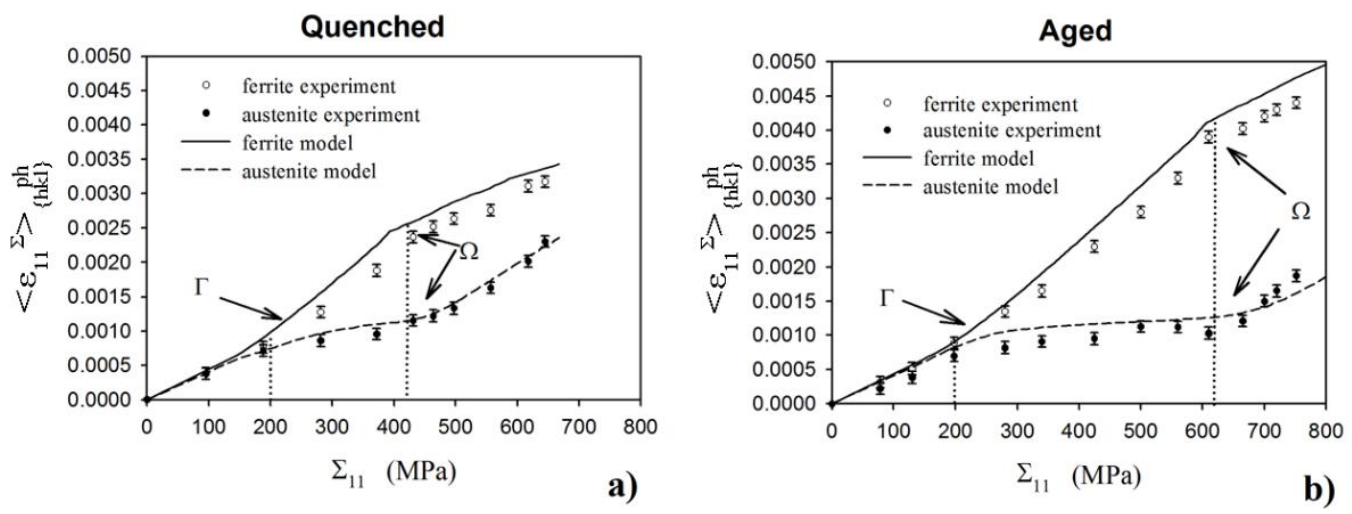

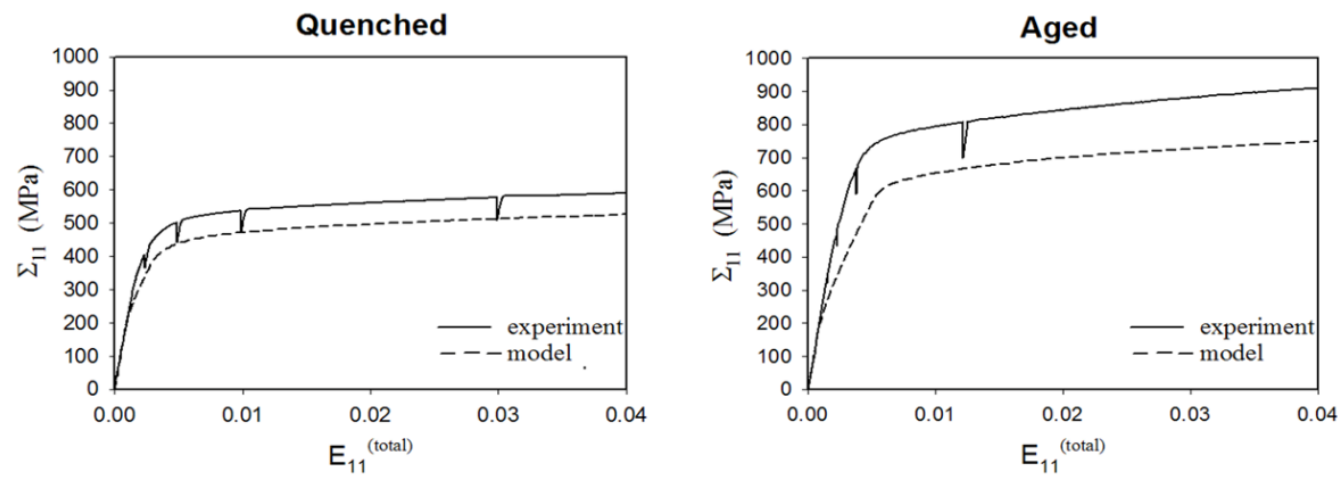

Fig. 15. Elastic lattice strain (a,b), corresponding to the applied stress $\Sigma_{11}$, measured by neutron diffraction using 111 reflection for austenite and 110 reflection for ferrite and results of the mechanical tensile test $(c, d)$. The experimental results are compared with model prediction.

\subsection{Plastic behaviour of the phases in duplex stainless steel}

One studied material was austeno-ferritic stainless steel (UR45N), containing approximately $50 \%$ austenite and $50 \%$ ferrite, obtained by continuous casting and subsequent hot rolling. The elastic lattice strains were measured in situ during the uniaxial tensile and compressive loadings using the time of flight (TOF) neutron diffraction (ENGIN-X instrument at the ISIS spallation neutron source at Rutherford Appleton Laboratory, UK). The experimental setup consisted of two detector banks which were centred on the horizontal scattering angles of $2 \theta=90^{\circ}$ (Fig.13). The detectors measured time-resolved spectra, each Bragg peak being produced by a reflection from a different family of $\{h k l\}$ planes (Fig. 14). The measurements were made at a series of controlled strains after the self-stabilisation of the load applied to the specimen (i.e. UR45 quenched sample). Inter-planar spacings along the loading direction were determined, while the sample strains were held constant during the measurement intervals. The relative elastic lattice strains were calculated with respect to the initial inter-planar spacings for different $\{h k l\}$ reflections in the direction of the applied load:

$$
<\varepsilon_{11}>_{\{h k l\}}=\frac{\left\langle d^{\Sigma}>_{\{h k l\}}-<d^{0}>_{\{h k l\}}\right.}{<d^{0}>_{\{h k l\}}}
$$

Where:

$<d^{\Sigma}>_{\{h k l\}}$ and $\left\langle d^{0}>_{\{h k l\}}\right.$ are the inter-planar spacings measured for the sample with and without external load.

The results of the self-consistent model were fitted to the experimentally determined lattice strain (Fig. 16a), as well as to the macroscopic curves (Fig. 16b) [2]. Single crystal elastic constants, measured crystalographical textures and initial stresses in both phases were used as the input data. It was found that the theoretical results confirm the evolution of the lattice strains in both phases and important information can be obtained from the applied analysis. The values of the critical shear stresses (and, consequently, of the yield stresses) were determined from the positions of characteristic thresholds on the vs. $\Sigma_{11}$ plots (Fig.16 a), i.e. $\Gamma$ determines yield stress for the austenitic phase, while the position of $\Omega$ depends on the yield stress of the ferritic phase. Thus, austenite is softer than ferrite in the studied duplex steel. On the other hand, it was found that the hardening of the austenitic phase was stronger compared with ferrite. 

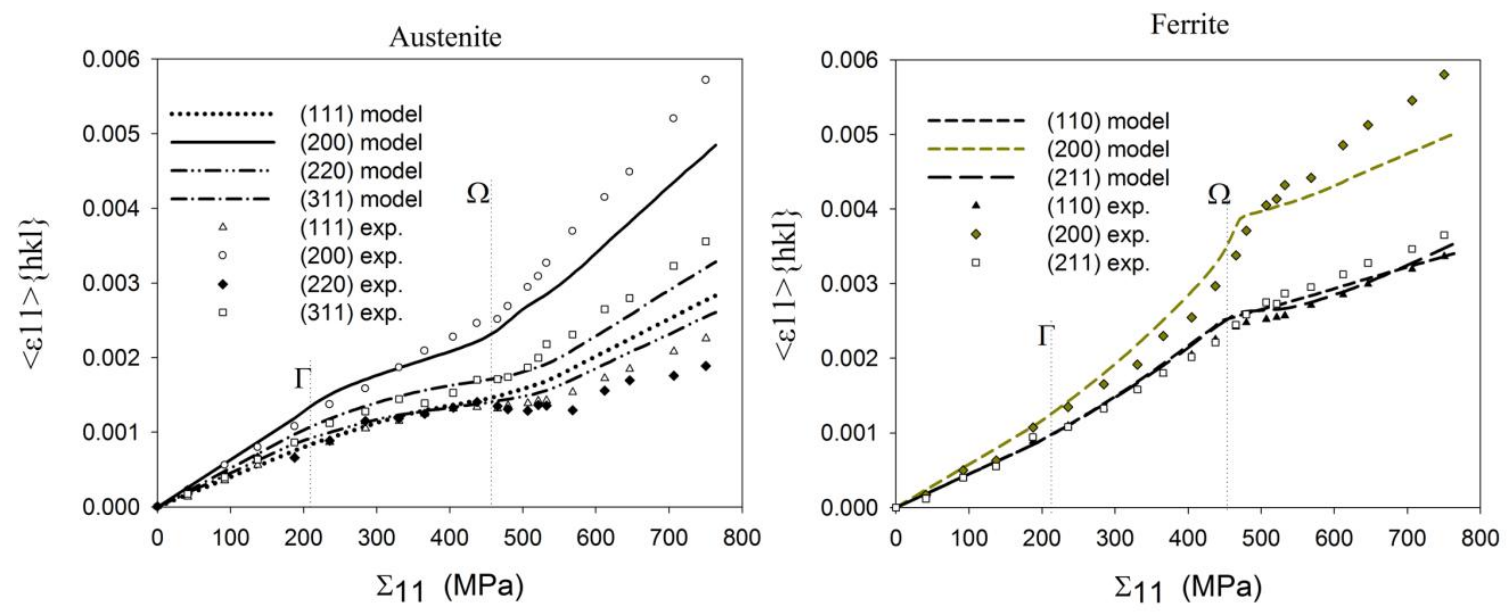

a)

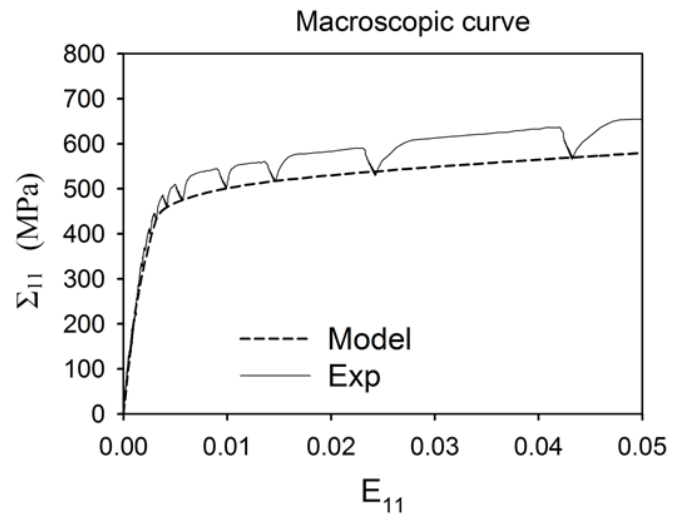

b)

Fig. 16. Elastic lattice strain (a) corresponding to the applied stress $\Sigma_{11}$, measured by neutron diffraction for austenite and ferrite and results of the mechanical tensile test (b).

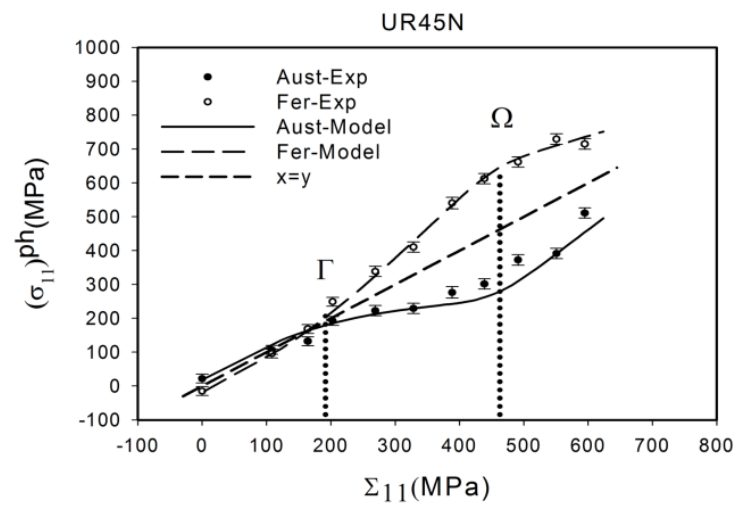

Fig. 17. Phase stresses corresponding to the applied stress $\Sigma_{11}$, measured by $X$-ray diffraction for austenite (111 reflection) and ferrite (110 reflection).

The results obtained by neutron measurements were also confirmed using X-ray diffraction (i.e., the standard $\sin ^{2} \psi$ method during in-situ tensile test). The phase stresses in function of macrostress $\Sigma_{11}$ were determined in each phase, independently. The theoretical results (selfconsistent model) agree very well with the experiment (Fig. 17) [3]. 


\section{RESULTS OF PREVIOUS EXPERIMENTS}

4.1. Measurements of the stress gradient around the rivets with $X$-radiation, extensometers and FE modeling

The specimen geometry is presented in Fig. 18. The specimen consisted of two sheets of PA7 aluminum alloy, which is the Polish equivalent of 2024T3 alloy, riveted with a press. The sheets were $1.27 \mathrm{~mm}$ thick and bare: without any protective layer such as cladding or an anodized layer (except for a thin layer of technological cladding approximately a few micrometers thick).

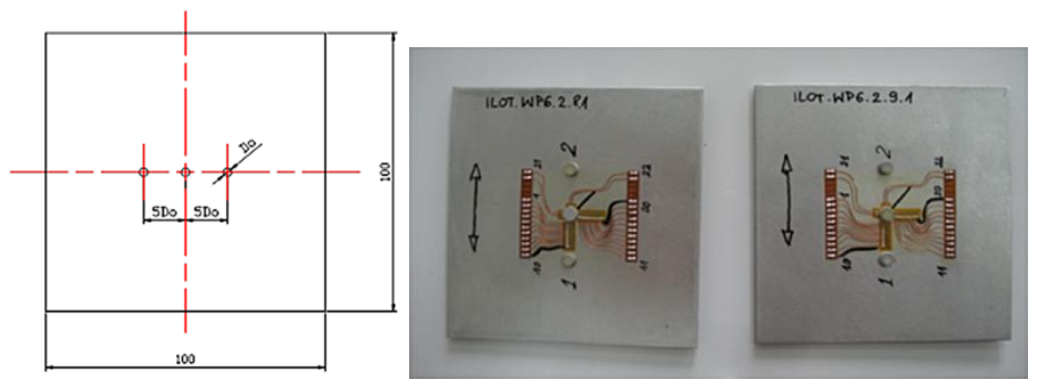

Fig. 18. Geometry of specimen for residual stress gradient measurements.

The strain gauges experiment was performed with the strip gauge patterns. Two different strain gauges were used - one of them measured tangential stresses while the other radial stresses. It was deposited on the edge of the hole for the rivet due to the high strain gradient - during riveting the nearest strain gauge on the chain was destroyed. The measurement was performed during the riveting process where the riveting force was controlled. The central rivets were riveted with a force giving the ratio between the driven rivet head and the rivet shank equal to 1.55. The riveted sample with strain gauges mounted is shown in Fig. 20, and the example of strain measurement during riveting is presented in Fig. 19. Two types of gauges were used: strip and micro gauges. The Vishay EA-13-XXXXX-120 strain gauges were employed (XXXXX stands for a gauge type). Strip miniature gauges contained ten gauges, each with a length of $0.51 \mathrm{~mm}$, and were located outside the driven head. They worked during the whole riveting process. Additionally, two micro strain gauges with a length of $0.38 \mathrm{~mm}$ were applied very close to the rivet hole, in the area which was under the driven head after the riveting process. The gauges recorded strains until they were destroyed by the driven head [4].

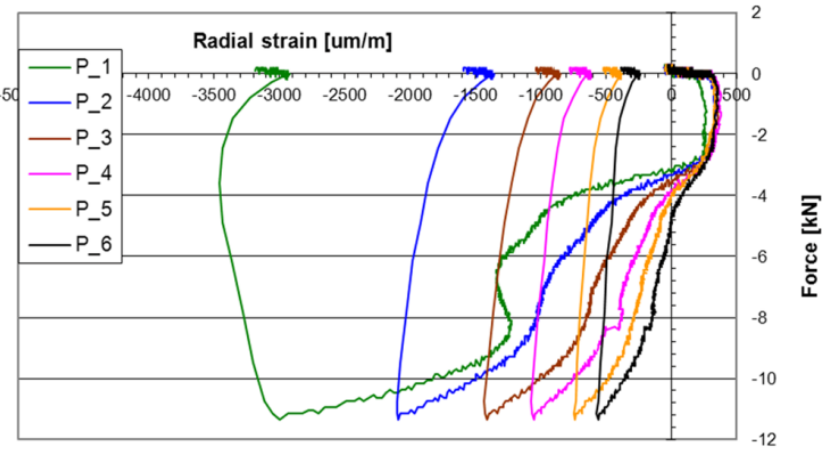

Fig. 19. Example of strain gauges measurement results during the riveting process.

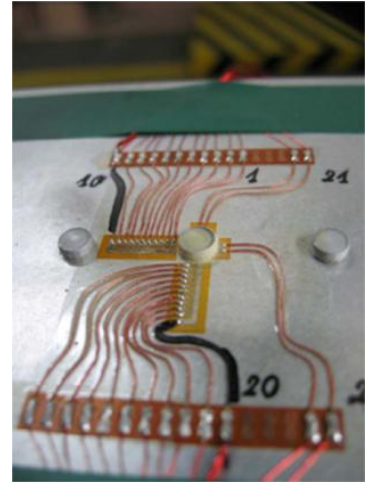

Fig. 20. Riveted specimen with strain gauges.

In finite element modeling, the axisymmetric models were developed. Each model consisted of six contact bodies; three deformable and three rigid ones and about 2, 000 linear elements. The material's isotropic elasto-plastic models, developed based on the monotonic test of specimens cut 
from sheet, and rivets were used. The stick-slip Coulomb model of friction was selected. Only the central rivet was taken into account (outer rivets were neglected). Previous analysis of the solid and axisymmetric models has indicated that this was a reasonable simplification. During the whole analysis the hold-on did not move. The press punch was able to move only in the axis direction and was controlled by force [4].

Exemplary results of diffraction measurements compared with strain gauges measurements and finite elements modelling are presented in Fig. 21.

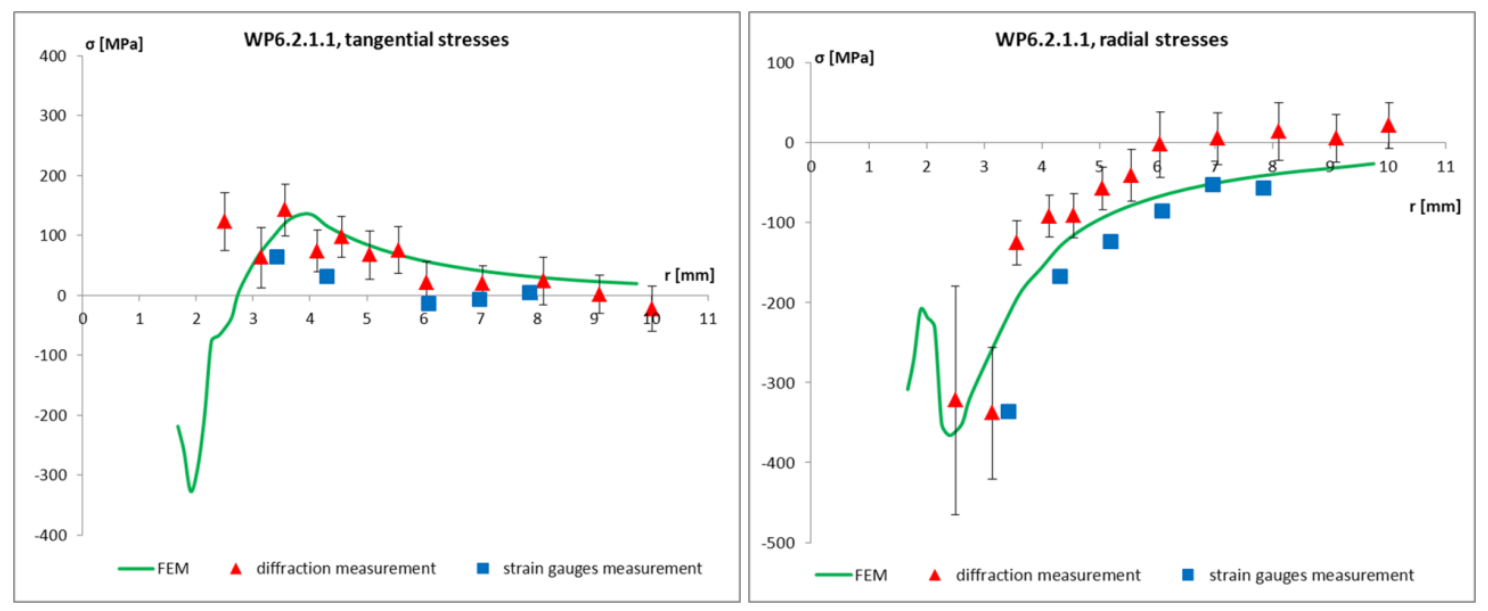

Fig. 21 Tangential and radial stress gradient around countersunk rivet without compensator. Comparison of diffraction, strain gauges measurements and FE modeling.

Good agreement betweenthe stress measurement results obtained with different techniques (Xray diffractometry and strain gauges) and the FE modeling gives strong evidence ofs the suitability of the applied methodology of X-ray stress measurements. The substantial procedure, to obtain a goodness of fit of these results was a subtraction of the initial stress state - his value was measure in a big distance from the rivet. The procedure followed in order to obtain a good fit of the results involved the subtraction of the initial stress state - its value was measured at a considerable distance from the rivet. Unfortunately, the experimental confirmation of the FEM analyses near the rivet shank was not possible because of the presence of the driven rivet head and a lower measurement resolution in case of X-rays and the strain gauges measurements. Unlike the $\mathrm{X}$-ray stress and strain gauges, the modeling was possible to perform - the strain gauge positioned nearest the rivet shank was destroyed during the riveting process. Unfortunately, the region most interesting for research is the area in the vicinity of the rivets - because of the expected highest stress gradient and because of the location of fatigue cracks. The issue of the stress modeling validation is planned to be researched in the future.

\subsection{Determination of local stress tensor}

A new method of measuring stresses for selected orientations of polycrystalline grains was demonstrated [5]. The new methodology is based on the measurements of lattice strains for several $h k l$ reflections and for different orientations of the sample during in situ tensile testing. The experimental data were analysed using the crystallite group method [6-8]. Measurements performed with a 6T1 diffractometer (LLB, Saclay) allowed researchers to determine the full stress tensor for several orientations of grains under applied uniaxial load (to find six components of the stress tensor, the lattice strains must be measured for at least six orientations of the scattering vector with respect to the sample).

The aim of this experiment was to determine the evolution of the stress tensor for preferred orientations of grains in duplex steel during tensile testing. Stresses for these orientations 
(i.e. $\{001\}\langle 011\rangle$ in ferrite and (i.e. $\{001\}\langle 100\rangle,\{011\}<211\rangle$ in austenite) can be calculated from strains measured for different orientations of the scattering vector (poles) with respect to the sample. This can be achieved by changing the sample orientation in Eulerian cradle and selecting $h \mathrm{kl}$ reflections by choosing appropriate values of $2 \Theta$ scattering angle.

The inter-planar spacings were measured with a wavelength of about $1.159 \mathrm{~A}$ and a slit aperture of $10 \times 10 \mathrm{~mm}^{2}$ (6T1 diffractometer) for the bone shape sample (cross section $4 \times 4 \mathrm{~mm}^{2}$ in the gauge part) under different loads applied by the tensile machine. Relative lattice strains were calculated with respect to the initial inter-planar spacings measured when the load was not applied to the sample (see eq. 7). To analyse the experimental results, the crystallite group method was usein order to calculate stresses related to the measured strains according to the relation:

$$
<\varepsilon(\phi, \psi)>_{\{h k l\}}=\gamma_{3 i} \gamma_{3 j} s_{i j k l} \sigma_{\mathrm{kl}}
$$

Where:

$\sigma_{\mathrm{k}}$ are the components of stress tensor for given orientation $\{\mathrm{uvw}\}<\mathrm{mno}>$ related to the set of strains $\langle\varepsilon(\phi, \psi)\rangle_{\{h k l\}}$ measured in the directions of scattering vector, $s_{i j k l}$ are the single crystal compliances calculated from stiffnesses and transformed to the sample frame, and $\gamma_{3 i}$ are the direction cosines transforming the grain strains into the direction of scattering vector.

The dependence of six stress components determined according to eq. 8 for three studied orientations is shown in fig. 22 alongside the prediction of the elastoplastic model $[9,10]$. The theoretical result was adjusted to the experimental data and the plastic parameters were found for both phases of studied steel. The Voce law describing hardening process at the grain scale was used and the plastic parameters were defined for each phase of the steel separately, i.e.:

$$
\tau^{g r}=\tau_{0}^{p h}+\left(\tau_{1}^{p h}+\theta_{1}^{p h} \xi^{g r}\right)\left[1-\exp \left(-\frac{\theta_{0}^{p h}}{\tau_{1}^{p h}} \xi^{g r}\right)\right]
$$

Where:

$\tau_{0}^{(p h)}, \tau_{1}^{(p h)}, \theta_{0}^{(p h)}$ and $\theta_{1}^{(p h)}$ are the adjusting parameters defined independently for each phase $p h$, while the plastic shear strain $\vartheta^{(g r)}=\sum_{t} \gamma^{t}$ is defined as the sum of total shear strains $\gamma^{t}$ for all slip systems $t$ in the grain $g r$.

Analysing the plots presented in fig. 22 it can be seen that there is a significant difference in the stresses localised in different phases. The evolution of the stress component parallel to the applied load $\left(\sigma_{11}\right)$ reflects the behaviour of the phases. The ranges in which both phases are elastic and both phases are plastic can be easily distinguished. The positions of the thresholds precisely determine yield stresses for each phase, which can be easily read from the presented plots. These thresholds are used to adjust the parameters of the Voce law. It can be noted that all components of the stresses are very accurately predicted by the model in the purely elastic range.

It can be stated that the presented methodology for the experimental determination of local stresses for crystallites having preferred texture orientations has been positively tested. Using the presented methodology, the localisation of stresses on polycrystalline grains, used in the scale transition models, can be verified. It should be noted that the analysis of the experimental data by crystallite group method can only be performed for strongly textured samples exhibiting pronounced preferred texture orientations. 

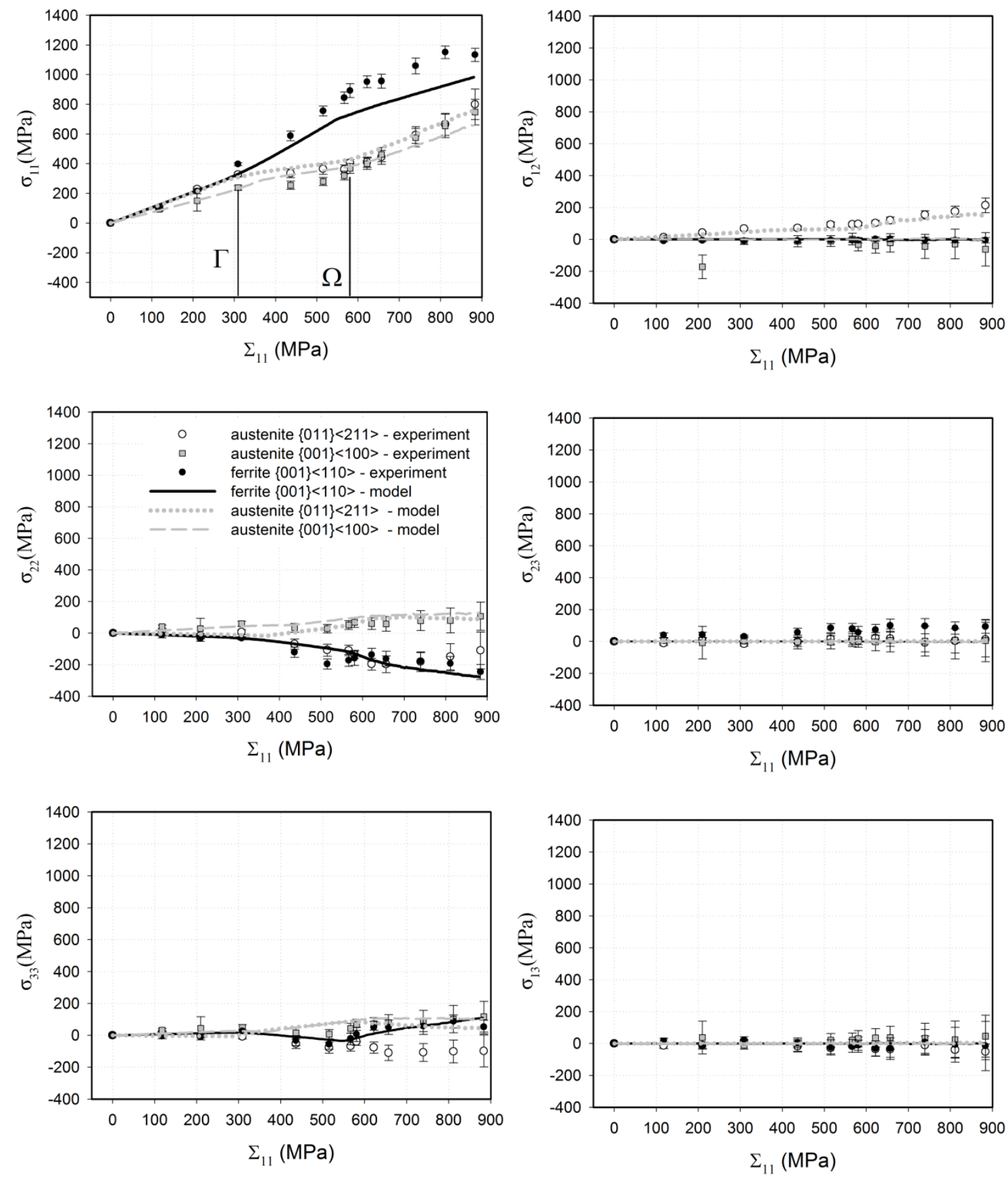

Fig. 22. Components of stress tensor localised on the group of grains having three studied texture orientations vs. applied macrostress. Experimental results obtained using crystalline group method (points) are compared with self-consistent prediction (lines).

\section{FUTURE RESEARCH}

\subsection{Concept and plan of the study}

Our past and future activities in general are focused on investigating of two-phases materials and working-out the methodology which will enable study properties of a every single phase and phenomena which take place in a mesoscopic scale. Our methodological aims are:

- development of the methodology of investigating of the polycrystalline materials properties in the mesoscopic scale and for different phases - using diffraction measurements, crystallographic models and FE calculations; 
- determination of stress concentration tensor with the experiment and the experiment results comparison with the theoretical predictions;

- model description of the damage phenomenon (mesoscopic scale and between phases);

- employing of the new worked out methodology for other two-phases materials (for example: metal matrix composite).

The detailed plan of our research is presented below.

\subsection{Small deformation and stress concentration tensor.}

\subsubsection{Initial research.}

New important information concerning small and large deformation of polycrystalline materials can be found by using neutron diffraction to measure lattice strains during tensile test. The differences in loading for groups of grains contributing to diffraction (selected by $h k l$ reflections) were observed during deformation history [2,11]. On the other hand, the evolution of the stress concentration tensor can be predicted using self-consistent model. This tensor describes differences between loadings for different grains. In the elastic range of deformation, localisation of the stresses depends on elastic anisotropy of crystallites, on orientations of their lattice and on the intergranular interactions. During yielding, plastic deformation gradually begins for different grains leading to load transfer between groups of grains $[2,11]$. In plasticity, stress concentration depends additionally on the anisotropy of plastic flow for grains having different orientations of slip systems with respect to applied load and on differences in resolved shear stresses. The above mentioned differences in stress localisation can be theoretically predicted for one or two phase materials.

To analyse loading history of different groups we assume that the plastic deformation does not depend on the rate of sample strain (time independent approach). Such assumptions is reasonable for the diffraction results which were obtained after stabilization of the applied macrostress for a given sample. In this case the concentration tensor $B_{i j k l}$ relating increase of strain at given grain $\Delta \sigma_{i j}$ and with the increment of the stress applied to the sample $\Delta \Sigma_{k l}$ can be written in the following form:

$$
\Delta \sigma_{i j}=B_{i j k l} \Delta \Sigma_{k l}
$$

The first aim is to determine the $\mathrm{B}_{\mathrm{ijkl}}$ for different orientations of grains within one or two phase materials grains. We propose a new method to analyze neutron and $\mathrm{X}$-ray diffraction results obtained during in-situ tensile test. In this method differential history of measured lattice strains in function of applied stress is presented and compared with model calculations for groups of grains selected by different $h k l$ reflections. As the result of comparison between experimental and theoretical data, the main component of stress concentration tensor can be determined. At first the study was performed for one phase austenitic steel for which preliminary measurements and interpretation has been performed. In future project stainless duplex steel will be investigated. We expect to observe significant difference between stress concentration in different phases.

5.2.2. in-situ measurement of strain in two directions during tensile test for duplex stainless steel with neutron diffraction at ISIS

The first experiment has been performed during tensile test for single phase austenitic steels using ENGIN-X instrument at the ISIS. As in previous experiment (see fig. 22), the lattice strains in function of applied load were determined. Next, the increments of the lattice strains $\Delta \varepsilon_{11}^{e l}$ (along applied load) vs. increments of macroscopic stress $\Delta \Sigma_{11}$. 


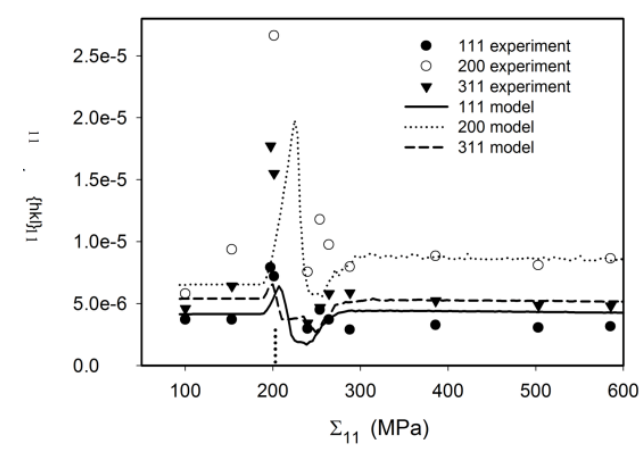

a)

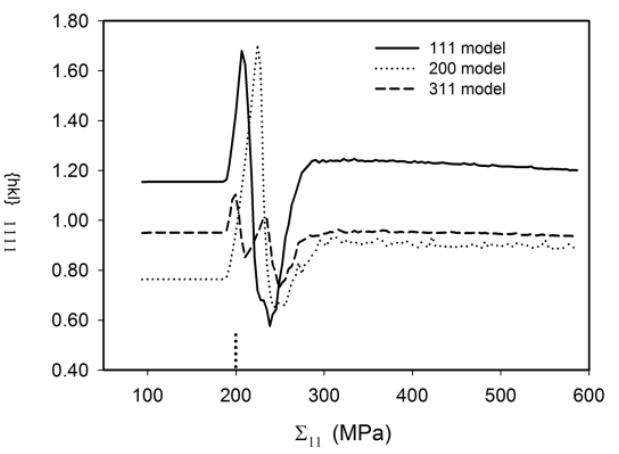

b)

Fig. 23 Derivative $\Delta<\varepsilon_{11}>_{\{h k l\}} / \Delta \Sigma_{11}$ (a) and average component $<B_{1111}>_{\{h k l\}}$ of stress concentration tensor (b) in function of macrostress $\Sigma_{11}$. Model prediction for parameters experimentally determined for AISI315L sample.

For each polycrystalline grain the increment of elastic strain can be expressed by increment of local stress using single crystal compliances $s_{k l i j}$, i.e.

$$
\Delta \varepsilon_{11}^{e l}=s_{11 i j} \Delta \sigma_{i j}
$$

Using eqs. 10 and 11 and after simple transformation the components of concentration tensor $B_{i j k l}$ can be related with lattice strain derivative determined from experiment or calculated by model:

$$
\frac{\left.\Delta<\varepsilon_{11}^{e l}\right\rangle_{\{h k l\}}}{\Delta \Sigma_{11}}=\left\langle s_{11 i j} B_{i j 11}\right\rangle_{\{h k l\}}
$$

The value $\left\langle s_{11 i j} B_{i j 11}\right\rangle_{\{h k l\}}$ bring us direct information about evolution of concentration tensor during deformation. Consequently, if the left side of eq. 12 is correctly predicted by model it means that also the evolution of $B_{i j 11}$ is well calculated.

Analyzing the results presented in fig. 23a, it can concluded that the elastic strain derivatives $\left(\Delta\left\langle\varepsilon_{11}^{e l}\right\rangle_{\{h k l\}} / \Delta \Sigma_{11}\right)$ calculated by model agree with the experimental ones. The best quantitative agreement is observed for range of sample deformation ( $\Sigma_{11}$ over 200MPa), when significant lattice strains are measured with good accuracy. An interesting results were obtained for the transition between elastic and plastic deformation $\left(\Sigma_{11}\right.$ about $\left.200 \mathrm{MPa}\right)$. In this case both model and experimental data show sharp instabilities of determined $\left.\Delta<\varepsilon_{11}^{e l}\right\rangle_{\{h k l\}} / \Delta \Sigma_{11}$ derivatives corresponding to transfer of the load between grains gradually entering plastic range of deformation. The instabilities of loadings are not proved quantitatively by experimental results, however qualitative agreement between model and experiment was found. As it was stated, the agreement of experimental and model values of $\left.\Delta<\varepsilon_{11}\right\rangle_{\{h k l\}} / \Delta \Sigma_{11}$ derivatives means that $B_{i j k l}$ tensor is correctly predicted. Thus, the average value of concentration tensor component $\left.<B_{1111}\right\rangle_{\{h k l\}}$ (calculated over diffracting grains volume for $h k l$ reflections) is presented in fig. $23 \mathrm{~b}$.

\subsubsection{Initial state of the duplex stainless steel - texture and microstructure.}

The initial state of the aged duplex steel UR45N (annealed during 1000 hours at a temperature of $400^{\circ} \mathrm{C}$ and then cooled in air) were already determined. 

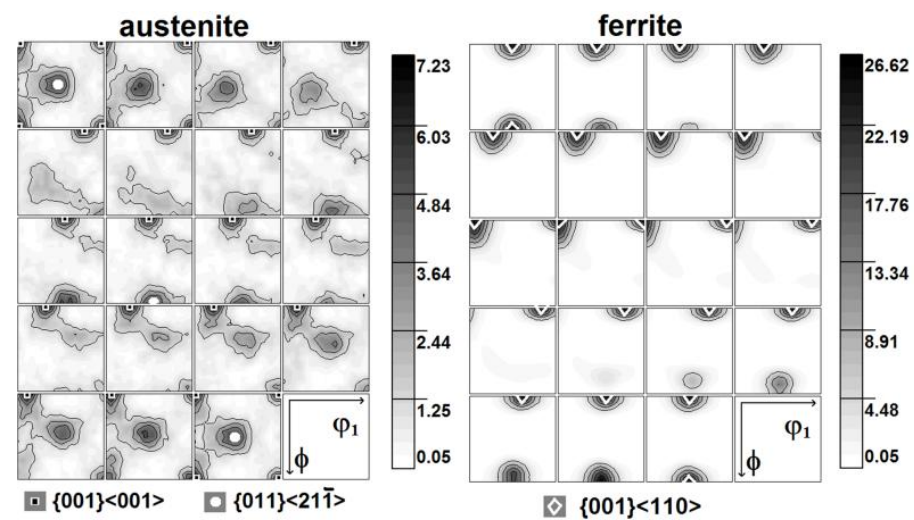

Fig. 24 The orientation distribution functions with the main preferred orientations are shown both phases of duplex steel UR45N. The sections through the Euler space with the step of $5^{\circ}$ along the $\phi_{2}$ axis are shown. The sample orientation is: $x_{1}\left\|R D, x_{2}\right\| T D$ and $x_{3} \| N D$ (where: $R D$-rolling, TD -transverse and ND - normal directions).

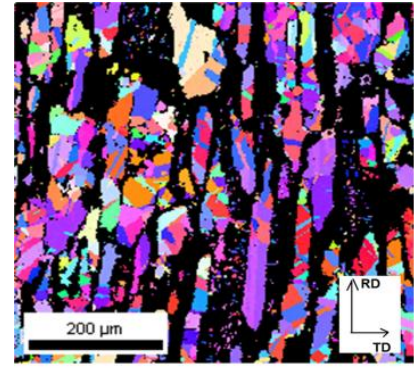

a)

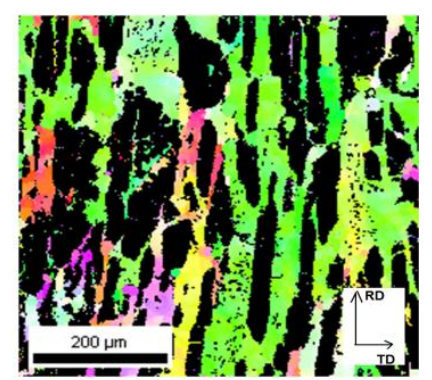

b)

Fig. 25 Microstructure of duplex stainless steels determined by the EBSD method. Orientations of (a) austenitic and (b) ferritic grains are shown in the selected phase by colors when the grains of unselected phase are black. The directions of rolling are indicated (i.e. $R D$-rolling, TD-transverse and NDnormal directions).

Crystallographic texture was measured using X-ray diffraction method. The orientation distribution functions (ODF) were calculated from experimental pole figures: $\{110\},\{200\}$ and $\{211\}$ for ferrite and $\{111\},\{200\},\{220\}$ for austenite. The main preferred orientations are indicated on the ODF sections (fig. 24). The microstructure of the studied steel was determined using the EBSD (electron back scattering diffraction) method. As shown in fig. 25 the austenitic phase creates islands elongated along the rolling direction and embedded in a ferritic matrix.

The initial state of the sample is important for model calculations in which the crystalographical texture, initial stresses and shape of grains are treated as the input data. To complete the input data for model calculations the initial stresses will be measured in this project.

\subsubsection{Planned research}

We plan to apply described above methodology to study elasto-plastic behavior of duplex steel. The results obtained for single phase steels will be compared with those obtained for one phase austenitic and ferritic steel. This comparison allow us to study influence of the phase interaction within multiphase materials. The measurements will be also performed for duplex steels having different chemical composition in order to study influence of composition of the material on the mechanical behaviour of the phases. Also, the in-situ tensile test in high temperature will be performed. The critical resolved stresses $\tau_{c r}$ and hardening parameters $H$ will be determined by comparison with self-consistent model and also the concentration tensor and its evolution will be determined. 
Moreover a new experimental method for study of plastic deformation at macroscopic and mesoscopic scales will be proposed. Last year a new possibility of stress measurements for selected orientations of polycrystalline grains was demonstrated [12]. The new methodology is based on the measurements of lattice strains for several $h k l$ reflections and for different orientations of the sample during in-situ tensile test (tensile machine is rotated in the Eulerian cradle). The experimental data were analyzed using crystallite group method [12]. Neutron measurements performed at 6T1 diffractometer (LLB, Saclay) will allow to determine full stress tensor for several orientations of grains under applied uniaxial load (to find six components of stress tensor, the lattice strains were measured for at least six orientations of scattering vector with respect to the sample).

The aim of our new method is to determine evolution of stress tensor for preferred orientations of grains in duplex steel during tensile test. It should be stated that the new methodology of stress measurement allow us to determine full stress tensor for grains having specific orientations directly from experiment without help of elastoplastic model. We expect to observe significant difference in the stresses localised on $\{100\}<011>$ orientation in ferrite and $\{001\}<100>$ and $\{011\}<21-1>$ orientations in austenite especially during yielding. Using texture we will check balance condition between components of stress tensor for main preferred orientations. Moreover, for the first time we will determine main components of stress concentration tensor. Finally, the evolution of $\sigma_{i j}$ and $B_{i j 11}$ will be compared with self-consistent model and plastic parameters $\left(\tau_{c r}\right.$ and $H$ ) will be found for preferred orientations. Moreover, knowing the stresses for given grain orientation, the Schmid relation used in the model can be verified.

In order to determine the components of $\mathrm{B}_{\mathrm{ij} 11}$ stress concentration tensor plan the following measurements:

- Using neutron and X-ray diffraction the inter-planar spacings $<d>_{\{h k l\}}$ vs. the $\sin ^{2} \psi$ will be measured.

- Using neutron method, the lattice strains $\left\langle\varepsilon_{11}\right\rangle_{\{h k l\}}$ Vs. $\Sigma_{11}$ plots and derivative $\Delta\left\langle\varepsilon_{11}^{e l}\right\rangle_{\{h k l\}} / \Delta \Sigma_{11}$ will be determined for stainless duplex steel and compared with single phase austenite and ferrite. Experiment will be also performed for duplex steels having different chemical compositions and also at higher temperature. The results will be analysed using selfconsistent model and FEM predictions.

- The principal components (and their increments) of the stress tensor $\sigma_{i j}$ in two phases will be determined during tensile test by using X-ray diffractometer. In this case the concentration tensor for each phase (austenite and ferrite) will be determined.

- The inter-planar spacings will be measured for the sample under different loads applied by tensile machine, rotated by Eulerian cradle (relative lattice strains are calculated with respect to the initial inter-planar spacings measured when the load is not applied to the sample). This experiment is planned at LLB, Saclay, France. The increments of the stress tensor $\Delta \sigma_{i j}$ will be determined using crystallite group method and the components of $\mathrm{B}_{\mathrm{ijkl}}$ tensor will be directly found for preferred crystal lattice orientations.

\subsection{Large deformation and damage}

\subsubsection{Initial research}

Nowadays, it is possible to develop numerical simulation for manufacturing processes in finite transformation. In order to improve the predictivity of crystallographic models and finite elements analysis (FEA), it is necessary to provide accurate constitutive models for mechanical behaviour. Such a methodology is now classical. The aim of the present work is to develop experimental techniques and data treatments to study damage mechanisms for metallic materials. Indeed, it is now well known that the coupling between damage and plasticity leads to an accurate description 
of ductile damage in metal forming [21,22]. We will study the opportunity to measure the mechanical consequences of damage in duplex steels, at different scales. Therefore, two scales have been considered and a particular experimental approach will be performed. Neutron diffraction coupled with in-situ tensile test can be used to study damage at mesoscopic scale. However, to extract damage occurring at macroscopic scale, it is necessary to take into account the necking phenomenon. In order to solve the problem of deformation heterogeneity, local strains were determined using the digital image correlation technique (DIC proposed by Peters [13]).

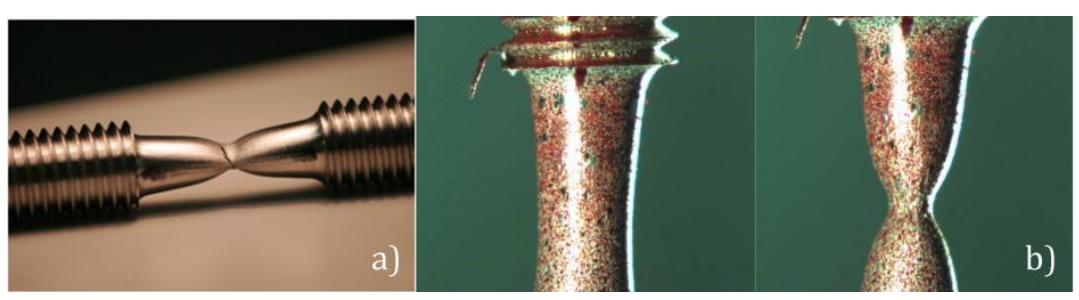

Fig. 26. Sample broken during in-situ neutron diffraction measurements (a) and pictures from two cameras (DIC method) corresponding at the end of the loading, close to the fracture time. "Pixels" on the pictures are due to speckle pattern treatment used for DIC calculations.

Recently, the experimental methodology based on neutron measurements and self-consistent crystallographic model were considerably developed for large strains including ductile damage effect. To achieve this, an original method for experimental data calibration was developed and tested [11] which must be used when the neutron measurements are performed within the neck, where heterogeneous deformation occurs. In analysis of the experimental data the results obtained applying DIC technique and FEM calculations are used.

The elastic lattice strains measured in-situ during the uniaxial tensile using TOF neutron diffraction (ENGIN-X, ISIS in Rutherford Appleton Laboratory, UK) together with macromechanical plots are shown in fig. 27 for the UR45 aged sample. Our calibration method has been applied for the presented data. It is striking that the evolution of grain stresses is almost linear with the external macrostress during plastic deformation (above the $\Omega$ threshold). This behavior is predicted by the model, even for non linear hardening behaviour, and exhibited experimentally. It extends to large strains until other phenomenon (relaxation) occures [11].
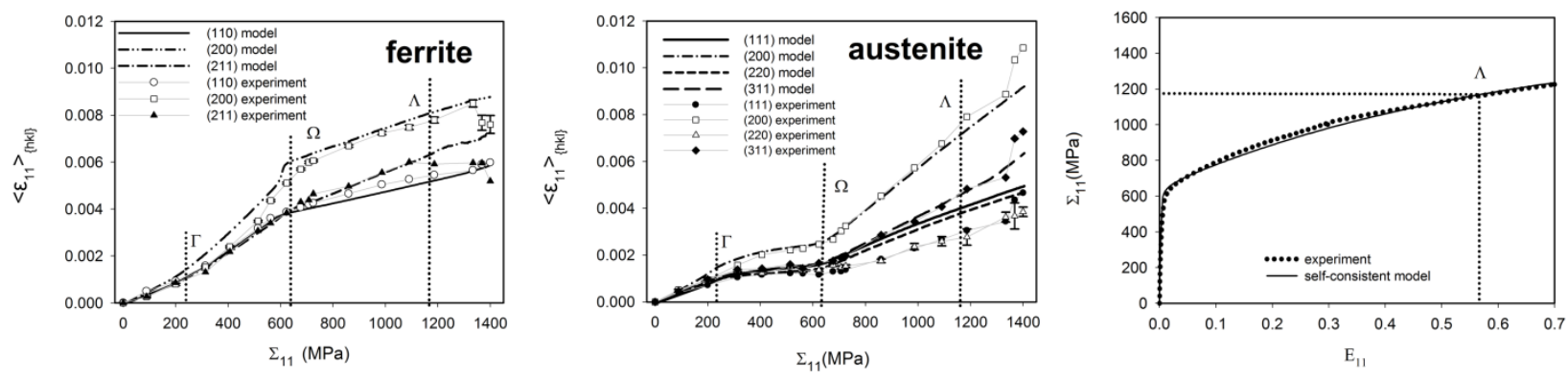

Fig. 27. Elastic lattice strain corresponding to the applied stress $\Sigma_{11}$, measured by neutron diffraction for austenite and ferrite and results of the mechanical tensile test. The experimental results are compared with model prediction

Methodology will be applied to study the effects of damage processes, which can be seen as a decrease of the measured lattice strains caused by the stress relaxation for the groups of grains selected by the given reflection. This decrease can be easily noticed in our recent experiment [11] due to a clear departure from the almost linear evolution of the lattice strains with respect to the macrostress during plastic deformation (fig.27). The possible micro-mechanical effects of damage 
were observed in the aged UR45N steel, showing a load transfer from damaging ferrite to nondamaging austenite. The microstructure study performed close to the fracture region of our UR45N aged sample confirms the hypothesis that the ferritic phase is responsible for damage initiation. The SEM image in fig.28 shows different processes initiating the damage process, i.e. decohesion occurring on slip system in the ferritic phase or at the grain boundary and in the presence of inclusions [11].

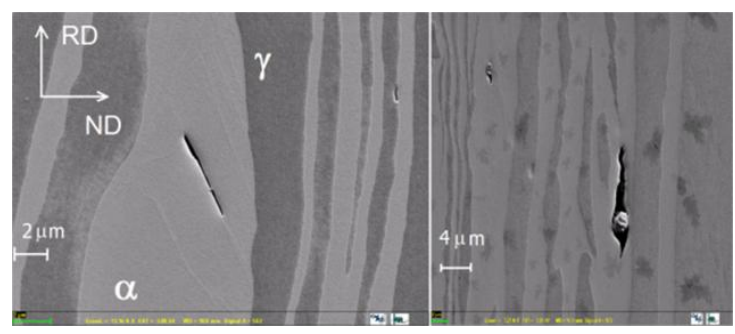

Fig. 28. Damage processes occurring on the slip plane or at the grain boundary (a) and caused by inclusions (b) are shown in SEM images.

\subsubsection{Planned research - damage modelling and the self-consistent model development}

To explain the phenomena observed recently in our experiments with neutron diffraction it is necessary to develop damage modelling in which the calculations in the macroscopic and mesoscale can be performed. In these models the damage process should be considered as the reason of stress relaxation in the ferritic phase. In this aim the self-consistent calculations can be applied in which constitutive equations, accounting for different kinds of hardenings weakly or strongly coupled with damage must be implemented.

In the present work, the continuous damage mechanism (CDM) approach, based on thermodynamics of irreversible processes [14,15] with state variables and state and dissipation potentials will be directly adapted to the grains scale. In the analysis the damaged state is related to the undamaged state by using energy equivalence principle $[14,15]$. Eventually, the multiscale self-consistent modelling scheme including damage effect will be developed. Basic formulation of the constitutive equations based on energy equivalence assumption have been already tested and published in [16].

The next important step is to develop the self-consistent model in order to predict the relaxation effects observed during neutron diffraction experiment for groups of grains. The theoretical calculations will allow us to understand physical phenomena occurring in the scale of polycrystalline grains, which are responsible for initiation of damage process (for example the level of stress localised on grains with particular orientations or belonging to particular phase, ductile damage occurring in one phase and causing instability of the material). Numerical algorithm describing damage process will be developed in the frame of self-consistent model used for many years by authors.

\subsection{Development the methodology for metal-matrix composites (MMC)}

\subsubsection{Initial research}

Modern technologies require materials with an unusual combination of properties that cannot be achieved in conventional alloys, ceramics or polymeric materials. To expand the range of conventional properties, a variety of composite materials have been developed that possess properties superior to each of the component phases; they are fabricated by introducing a reinforcement, usually a ceramic one, into metal or alloy.

Neutron diffraction was already used (LLB, Saclay) to measure the lattice strains in $\mathrm{Al} / \mathrm{SiCp}$ metal-matrix composite under an external load applied by bending while the sample was in-situ in the neutron beam [17]. The evolution of the internal stresses and of the critical-resolved shear 
stress during bending were predicted by elastoplastic models. It was found that the self-consistent model correctly predicts the distribution of stresses between the two phases of the $\mathrm{Al} / \mathrm{SiCp}$ composite. Also, it was shown in $[18,19]$ (LLB, Saclay) that after elastoplastic deformation the mismatch stresses determined for both phases relax for tensile as well as compressive deformation but this process does not correspond to the results of the self-consistent model. The micromechanisms operating in this case are quite complex, with relaxation occurring equally in both tensile and compression modes can be caused by microdamage process occurring on the Almatrix and $\mathrm{SiC}$-particle interfaces. It was shown, that plastic flow in the matrix has reduced the effect of thermal treatment of the material. This may have important consequences for applications of MMCs [18,19].

\subsubsection{Planned research}

The aim of present work is to study mechanical effects occurring between metal matrix and reinforcements during elatoplastic deformation of metal-matrix composite at relatively large deformations until fracture. In this study the MMC reinforced with ceramic particles will be studied. The first considered material will be the $\mathrm{Al} / \mathrm{SiC}_{\mathrm{p}}$ composite which was already investigated by authors of this work $[17,18,19]$. Next, the methodology will be applied and tested for composites based on the Ti matrix. In this case, however, the self-consistent model must be developed for hexagonal crystal symmetry.

As it has been shown in the previous sections, measurements already done for duplex steel ([1] LLB, Saclay and $[2,11,20]$ ISIS RAL, UK) clearly show the variation of lattice strains during elastic-plastic transition of both phases. In the case of the present experiment, we expect to observe the elastic-plastic transition for aluminium matrix and next the reduction of mismatch stresses due to microdamage process starting on the $\mathrm{SiC}-\mathrm{Al}$ interface. We propose to measure lattice strains for both phases separately using two separate diffraction peaks (for example 111 reflection of $\mathrm{Al}$ and also 111 reflection of $\mathrm{SiC}$ ) during in-situ tensile test. Lattice strains will be measured in the direction parallel and direction perpendicular to the applied load. We will determine the applied stress and sample strain for elastic-plastic transition of aluminium and for beginning of microdamage process or relaxation of mismatch stresses. The results will be compared with elastoplastic model in order to find parameters determining plastic deformation of $\mathrm{Al}$ matrix (critical resolved shear stress and hardening parameter). Finally, the evolution of stress concentration tensor will be determined.

\section{REFERENCES}

[1] Baczmański, A., Dakhlaoui, R., Braham, C., Wierzbanowski, K., Arch. Metall. and Mater., 53, 89-96 (2008)

[2] Dakhlaoui, R., Baczmański, A., Braham, C., Wroński, S., Wierzbanowski, K., Oliver, E.C. (2006). Acta Mater. 54, 5027 -5039.

[3] Dakhlaoui, R., Braham, C., Baczmański, A. (2007). Mater. Sci. Eng. A 444, 6-17.

[4] Kaniowski J.: Improving the Fatigue Performance of Riveted Joints in Airframes, research project report Nr EUREKA/59/2006, Institute of Aviation, Warsaw 2010.

[5] R. Dakhlaoui, V. Klosek, M.H. Mathon, B. Marini, Acta Mater. 58(2010) 499-509.

[6] P.F. Willemse, B.P. Naughton and C.A. Verbraak. Mater. Sci. Eng. 56 (1982) 25-37

[7] B.J. Ortner, J. Appl. Cryst. 22 (1989) 216.

[8] V. Hauk, "Structural and Residual Stress Analysis by Nondestructive Methods", Elsevier, Amsterdam, Lausanne, New York, a.o., (1997)

[9] P. Lipinski and M. Berveiller, Int. J. Plastic. 5 (1989) 149-172.

[10] P. Zattarin, A. Baczmański, P. Lipiński and K. Wierzbanowski, Arch. Metall. 45 (2000) 163184. 
[11] Baczmanski, A. , Le Joncour, L., Panicaud, B. , Francois, M. , Braham, C., Paradowska A. M., Wroński, S., Amara S. and Chirone R., J. Appl. Cryst., 44, (2011) 966-982

[12] Dakhlaoui, R. Klosek, V., Mathon, M.H., Marini, B. (2010) Acta Mater. 58 499-509.

[13] Peters, W.H. and Ranson, W.E. (1982). Opt. Eng. 21, 427-432.

[14] Saanouni, K., Abdul-Latif, A., Int. J. Plasticity 12 (1996) 1111-1121.

[15] Chaboche, J.-L. , Boudifa, M., Saanouni, K., Int. Journal of Fracture 137 (2006) 51-75.

[16] Panicaud, B., Saanouni, K., Baczmanski, A., François, M., Cauvin, L., Le Joncour, L., , Computational Materials Science, 50, (2011) 1908-1916.

[17] Baczmański, A., Levy-Tubiana, R., Fitzpatrick, M.E., Lodini, A., (2004). Acta Mater. 52, 1565-1577.

[18] Fitzpatrick, M.E., Withers, P.J., Baczmański, A., Hutchings, Levy, M.T. R., Ceretti M. and Lodini, A., Acta Mater., 50, 1031-1040 (2002)

[19] Levy-Tubiana, R., Baczmański, A. and Lodini, A., Mater. Sci \& Eng. A, 341, 74-86 (2003)

[20] Baczmański, A., Braham, C., Seiler, W.,(2003). Phil. Mag. 83, 3225-3246. 